\title{
Optimal Cognitive Access of Markovian Channels under Tight Collision Constraints
}

\author{
Xin Li, Qianchuan Zhao, Xiaohong Guan, Fellow, IEEE, and Lang Tong, Fellow, IEEE
}

\begin{abstract}
The problem of cognitive access of channels of primary users by a secondary user is considered. The transmissions of primary users are modeled as independent continuous-time Markovian on-off processes. A secondary cognitive user employs a slotted transmission format, and it senses one of the possible channels before transmission. The objective of the cognitive user is to maximize its throughput subject to collision constraints imposed by the primary users. The optimal access strategy is in general a solution of a constrained partially observable Markov decision process, which involves a constrained optimization in an infinite dimensional functional space. It is shown in this paper that, when the collision constraints are tight, the optimal access strategy can be implemented by a simple memoryless access policy with periodic channel sensing. Analytical expressions are given for the thresholds on collision probabilities for which memoryless access performs optimally. Extensions to multiple secondary users are also presented. Numerical and theoretical results are presented to validate and extend the analysis for different practical scenarios.
\end{abstract}

Index Terms-Cognitive radio, Dynamic spectrum allocation, Cognitive medium access, Markov decision processes.

\section{INTRODUCTION}

$\mathbf{W}$ E CONSIDER a hierarchical overlay cognitive network with $N$ parallel communication channels shared by primary and secondary users [1]. The Primary Users (PUs) communicate through dedicated channels, oblivious to the presence of Secondary Users (SUs). On the other hand, a secondary user (SU) transmits opportunistically by first sensing a candidate channel. Based on the sensing outcome, the SU will decide if and where to transmit. The SU must also obey certain collision constraints so that its transmission will not interfere the communication of PUs beyond the acceptable levels. The first such cognitive access scheme for slotted primary and secondary users was proposed in [2].

In this paper, we model the occupancy of PUs as independent continuous-time on-off Markov processes with "on" indicating that the channel is being used by a PU and "off"

Manuscript received 8 December 2009; revised 29 May 2010 and 13 September 2010. This work was supported by NSFC Grant (60574067, $60736027,61021063,61074034)$ and by the Programme of Introducing Talents of Discipline to Universities (the National 111 International Collaboration Project, B06002). The work of L. Tong was supported in part by the US Army Research Office MURI Program under award W911NF-08-1-0238 and the National Science Foundation under award CCF 1018115. A conference version of this paper appeared in the 2010 Intl. Conf. Communications.

X. Li, Q. Zhao, X. Guan are with the Center for Intelligent and Networked System, Department of Automation and TNLIST, Tsinghua University, Beijing 100084, China (e-mail: \{zhaoqc,xhguan\}@tsinghua.edu.cn).

L. Tong is with the School of Electrical and Computer Engineering, Cornell University, Ithaca, NY 14853, USA (e-mail: 1tong@ece.cornell.edu).

Digital Object Identifier 10.1109/JSAC.2011.110407. for the case when the channel is idle. This model is an approximation of existing wireless access applications (e.g. 802.11 WiFi) [3].

The aim of the SU is to maximize its throughput subject to collision constraints imposed by the primary users. For example, the network designer may want to assure the PUs that, whenever they transmit, the probability of colliding with an opportunistic SU is below a threshold, say, less than $1 \%$. With such a guarantee, the PU may be willing to allow cognitive transmissions if the PU is compensated accordingly.

Obtaining the optimal access policy for the SU appears to be intractable at the first glance. In particular, the optimal access policy includes both sensing and transmission policies, and the design of jointly optimal policies are in general not separable. Because the SU can only observe one of the available channels, the problem falls into the category of partially observable Markov decision process (POMDP) with constraints, for which there is no general practical solution available [4]. It seems that the optimal access policy will have complexity growing exponentially with respect to the number of channels.

\section{A. Main Results}

In this paper, we first consider the case when there is a single secondary user and show that, when the the collision constraints are tight, the optimal access policy can be implemented by a simple memoryless policy with linear complexity. Referred to as periodic sensing and memoryless access (PSMA), the policy senses channels in a round robin fashion and transmits with a certain probability as a function of the collision constraints and traffic statistics of the primary users. To establish the optimality of PS-MA, we take an indirect approach by comparing PS-MA with the optimal (clairvoyant) policy that assumes full spectrum observation. These two policies establish the lower and upper bounds on the performance of the optimal policy, respectively. A main results of this paper is to show that, when the collision constraints are tight, the lower and upper bounds match. In addition, we also provide analytical characterizations of the critical thresholds for collision constraints under which PS-MA is optimal.

Next we generalize the single (secondary) user access policy to a multiuser setting. To this end, the structure of PS-MA lends itself to a natural extension for cases when multiple SUs are competing for spectrum opportunities. We present two access strategies. The first relies on orthogonalizing SUs by separating the phase of their channel sensing. Here the only modification required is a proper scaling of the transmission 
probabilities. Under tight collision constraints, we again obtain an analytical characterization of the performance. We show that, as the number of SUs increases, PS-MA becomes more efficient. The second policy is an application of CSMA random access techniques to PS-MA.

It seems surprising that a suboptimal (periodic) sensing policy with memoryless transmissions (PS-MA) can match to the case when all channels are sensed simultaneously. When full spectrum sensing is used, more opportunities are discovered. To meet tight collision constraints, however, the transmissions of SU must be limitted by lowering its transmission probabilities. Thus not all transmission opportunities can be utilized. The generally suboptimal policy PS-MA, on the other hand, discovers only a fraction of spectrum opportunities, which allows the SU transmitting more aggressively. The tight collision constraints play an equalizing role that leads to same throughput performance between the algorithm with full spectrum sensing and that using periodic sensing.

\section{B. Related Work}

We will restrict our comments on related work to the problem of cognitive access in a hierarchical network of primary and secondary users. In this context, we refer to a recent survey by Zhao and Sadler [1]. Related work in a broader context can be found in [5].

The joint design of sensing and transmission policy that maximizes the throughput of a cognitive SU subject to collision constraints is difficult in general, and it becomes tractable only when certain structures are imposed on the primary and secondary users. In [2], [6], Zhao et al. consider the case when all users follow a slotted transmission structure: when a PU has packets to transmit, it will do so at the beginning of the slot. Imposing a slotted structure simplifies the problem considerably, thanks to the separation principle [7] and the optimality of myopic policies [8], [9].

The problem of cognitive access of multiple continuoustime Markovian channel is first considered in [10], [11]. The authors simplify the problem by restricting the sensing policy to a periodic sensing scheme, which changes the problem from a partially observable Markov decision process with constraint to a constrained finite state Markov decision process. The resulting algorithm, referred to as optimal spectrum access with periodic sensing (PS-OSA), can be obtained from a linear program. Other related work assuming un-slotted PUs can be found in [3], [12], [13], [14].

The fundamental limit and structure of cognitive access of a single continuous-time Markovian channel is investigated in [13], [15] where the authors consider the optimal transmission policy with arbitrarily small sensing and transmission periods. The optimal transmission is probabilistic. For the single channel case, PS-MA reduces asymptotically to the access policy considered in [13] as the duration of the transmission period approaches to zero.

The existing result most relevant to this paper is [11] where the two algorithms (FO-OSA and PS-MA) analyzed here were first presented. It is therefore necessary to delineate the contribution in this paper beyond that in [11]. The full observation and optimal spectrum access (FO-OSA) policy was presented in [11] without performance analysis, and it was used as a throughput upper bound in simulations. The analysis of this policy presented in this paper is new and nontrivial.

The PS-MA policy that serves as a performance lower bound is first introduced in [10] and is independently considered by Akbar and Tranter [16]. While the approach presented in [16] has the same periodic sensing structure, it does not consider constraints on collisions. The PS-MA policy presented here is also slightly different from that in [11]. In particular, the policy presented in [11] has a bias, which is removed in this paper. The performance of PS-MA has not been analyzed in the past and its optimality is first presented in this paper.

There has been limitted published work on the cognitive access of multiple channels when multiple secondary users are involved until recently. From a multiaccess point of view, closest to our approach is the work of Wang et. al. [17] in which an ALOHA style protocol was proposed for a system similar to one considered here. There are some work on the multiple secondary users for systems where primary and secondary users are both slotted [18], [19]. These results are very different from the ones considered in this paper in the models assumed and techniques used. A substantial generalization of the results here to the multiuser setting can be found in [20].

\section{Model, Performance, and Optimality}

Assume that there are $N$ parallel channels (indexed from 0 to $N-1$ ) available for transmissions by the PUs. The occupancy of the $N$ channels is modeled by $N$ independent continuous-time Markov chains. In particular, each channel has two states with idle $\left(X_{i}=0\right)$ and busy state $\left(X_{i}=1\right)$, respectively. The holding times are exponentially distributed with known parameters $\lambda_{i}^{-1}$ for the idle and $\mu_{i}^{-1}$ for the busy states, respectively. The state transition rate matrix ( $Q$-matrix) under the continuous-time Markov process is given by

$$
Q_{i} \triangleq\left(\begin{array}{cc}
-\lambda_{i} & \lambda_{i} \\
\mu_{i} & -\mu_{i}
\end{array}\right), \quad i=0,1, \ldots, N-1 .
$$

The stationary distribution of the $i$ th PU process can then be determined as

$$
v_{i}(0)=\frac{\mu_{i}}{\lambda_{i}+\mu_{i}}, \quad v_{i}(1)=\frac{\lambda_{i}}{\lambda_{i}+\mu_{i}} .
$$

The set of admissible access policies $\mathscr{P}$ for an SU is defined as follows. We assume that the SU employs slotted transmissions. At the beginning of each slot, the SU chooses one of the $N$ channels to sense and makes a decision to transmit in one of the $N$ channels or not to transmit at all. Thus each access policy is the product of a sensing policy and a transmission policy, and the corresponding action space of the SU is a product of sensing and transmission spaces $\mathcal{S} \times \mathcal{A}$ where $\mathcal{S}=\{0, \cdots N-1\}$ is the set of channels to sense and $\mathcal{A}=\{-1,0, \cdots N-1\}$ the set of transmission actions with $i$ indicating the transmission on the $i$ th channel and -1 indicating no transmission.

Fig 1 illustrates a realization of an access policy of a single SU. If the SU transmits on a particular channel and it does not collide with the PU during its transmission, then the SU 


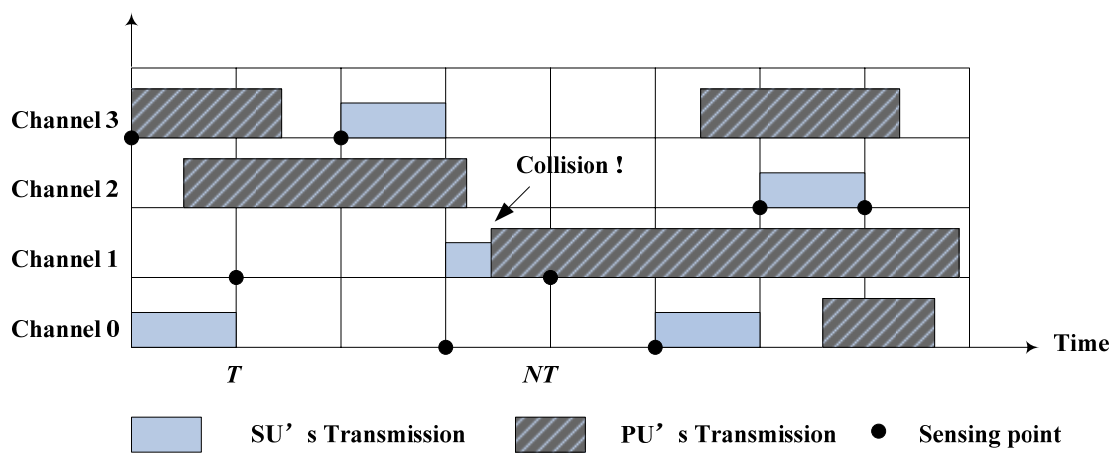

Fig. 1. Illustration of a general admissible access protocol. The transmissions are in general probabilistic. Collisions may happen over channels that are sensed idle.

receives a reward of 1 (successfully transmitted packet). If a collision happens, the SU receives no reward, and the collision is counted against the $\mathrm{SU}$ as an instance of violating the collision constraints. Note that for continuous-time Markov channels, if the SU transmits on channel $i$ that is idle initially, because the PU may start the transmission at any time, the expected reward received by the $\mathrm{SU}$ is $\exp \left(-\lambda_{i} T\right)$ where $\lambda_{i}$ is the rate that the channel $i$ stays idle and $T$ the slot duration. When multiple SUs are involved, an SU receives a reward if it does not collide with PU or another SU.

We use the average throughput as the performance metric. For a fixed policy $\pi$, let $R_{k}^{\pi}$ be the expected reward received in the $k$ th slot, the average throughput of $\pi$ can be defined by

$$
J(\pi)=\lim _{K \rightarrow \infty} \frac{1}{K} \sum_{k=1}^{K} E_{\pi}\left(R_{k}^{\pi}\right),
$$

where the expectation is taken over the probability distributions induced by $\pi$.

We will impose collision constraints on SU's transmissions, using the conditional collision probability as the metric. Specifically, we denote $C_{i}(\pi)$ as the probability, conditional on the $i$ th PU transmitting, that policy $\pi$ leads to a collision between the SU and the $i$ th PU. When multiple SUs are present, collisions between the PU and all SUs are counted.

We define the optimal single user access policy $\pi^{*}$ as the solution of the following constrained optimization

$$
\max _{\pi \in \mathscr{P}} J(\pi) \text { subject to } C_{i}(\pi) \leq \gamma_{i}, \quad i=0, \ldots, N-1,
$$

where $0 \leq \gamma_{i} \leq 1, i=0, \ldots, N-1$ are given constants representing tolerable collision levels. When multiple SUs are involved, the situation is more complicated. See [20]. We consider here only the sum-rate formulation by replacing $J(\cdot)$ in (3) with the sum of the average rewards from all users. Note that the collision constraints in (3) remain the same.

\section{Single User Cognitive Access Policies}

We first consider the case involving a single SU who learns transmission opportunities by sensing one channel at a time and deferring the discussion of the multiuser case to Sec. V.

There is, unfortunately, no general tractable solution to the constrained Partially Observable Markov Decision Processes (POMDP) in (3). We take an indirect approach by considering some simpler policies that give the lower and upper bounds on the performance.

For the performance upper bound, we consider a clairvoyant policy that assumes full spectrum sensing. Referred to as optimal spectrum access with full observation (FO-OSA), the policy $\pi^{\mathrm{FO}}$, first introduced in [11] as a benchmark for comparison, maximizes the SU throughput subject to the same collision constraints as that for $\pi^{*}$. Since it uses more information allowed by $\pi^{*}$, we naturally have $J\left(\pi^{*}\right) \leq J\left(\pi^{\mathrm{FO}}\right)$.

For the performance lower bound, we consider a policy referred to as the Periodic Sensing with Memoryless Access (PS-MA), denoted by $\pi^{\mathrm{MA}}$. PS-MA is a policy that senses each channel periodically (round robin) and it makes the transmission decision based on the current sensing outcome. PS-MA belongs to the set of admissible policies and is in general suboptimal. PS-MA was first introduced in [11], again as a numerical benchmark. The version presented here is a slight improvement over that in [11]. In this case, we clearly have $J\left(\pi^{\mathrm{MA}}\right) \leq J\left(\pi^{*}\right)$.

The main result of this paper is to establish a threshold condition on collision constraints such that $J\left(\pi^{\mathrm{MA}}\right)=J\left(\pi^{\mathrm{FO}}\right)$. Because $J\left(\pi^{\mathrm{MA}}\right) \leq J\left(\pi^{*}\right) \leq J\left(\pi^{\mathrm{FO}}\right)$, we can claim that the simple policy PS-MA is in fact optimal. In this section, we give the mathematical formulations that define $\pi^{\mathrm{MA}}$ and $\pi^{\mathrm{FO}}$.

\section{A. Full Observation with Optimal Spectrum Access: $\pi^{F O}$}

By full observation we mean that all channels are sensed simultaneously at the beginning of every time slot. Policies based on full observation are clairvoyant policies because the set of admissible policy $\mathscr{P}$ allows only sensing a single channel.

The FO-OSA policy is the optimal policy based on the full channel state information $X(k)=x, x \in\{0,1\}^{N}$ in each time slot $k$, i.e., the SU chooses the action $i \geq 0$ based on the observation $x$ with the probability $\beta_{i}(x)$ transmitting on channel $i$. The immediate reward when channel $i$ is used for transmission is given by

$$
g(x, i)=\left\{\begin{array}{cc}
\exp \left(-\lambda_{i} T\right), & x_{i}=0 ; \\
0, & x_{i}=1,
\end{array}\right.
$$

which is also the probability of collision-free transmission by the SU on channel $i$ conditioned on $X(k)=x$ and the SU does transmit on channel $i$. Equivalently, $g(x, i)$ is also the average 
successful transmission by the SU when $X(k)=x$ and $\mathrm{SU}$ chooses to use channel $i$.

Let $f(x)$ be the stationary distribution of the state vector $X(k)$. It is easy to show that the optimal access policy is stationary, and it is fully specified by its (conditional) transmission probability $\beta(x) \triangleq\left(\beta_{i}(x)\right)$. The optimal policy given full observation is therefore obtained by the optimal choice of $\{\beta(x), \forall x\}$ subject to collision constraints. Specifically, the probability vector $\beta=(\beta(x))$ corresponding to $\pi^{\mathrm{FO}}$ can be determined by solving the following linear program:

$$
\max _{\beta \in[0,1]^{N \times 2^{N}}} \sum_{x \in\{0,1\}^{N}} f(x) \sum_{i=0}^{N-1} g(x, i) \beta_{i}(x)
$$

subject to

$$
\begin{gathered}
\sum_{x \in\{0,1\}^{N}} \frac{f(x)(1-g(x, i)) \beta_{i}(x)}{1-v_{i}(0) \exp \left(-\lambda_{i} T\right)} \leq \gamma_{i}, \quad \forall i \\
\beta_{i}(x) \geq 0, \quad \sum_{i=0}^{N-1} \beta_{i}(x) \leq 1, \quad \forall x,
\end{gathered}
$$

where in (5), $g(x, i) \beta_{i}(x)$ is the probability of the SU successfully transmitted on channel $i$ conditioned on $X(k)=x$, and the expression in (5) is the average success rate of transmission by the SU. For the constraint in (6), the denominator is the probability of the PU on channel $i$ transmitting, $(1-g(x, i)) \beta_{i}(x)$ the probability of $\mathrm{PU}$ and $\mathrm{SU}$ transmitting simultaneously conditioned on $X(k)=x$, thus the term inside the summation is the joint probability of $X(k)=x$ and collision occurs conditioned on the PU on channel $i$ transmits in slot $k$. Note that the inequality in (7) allows the possibility that SU keeps silent even when opportunities of transmissions exist.

\section{B. Memoryless Access with Periodic Sensing: $\pi^{M A}$}

The policy PS-MA, first introduced in [11], decouples sensing and access. PS-MA senses the channels in an increasing order. In particular, in slot $k$, the SU senses channel $q=k \bmod N$. Policy PS-MA has no memory, and the transmission will only depend on the current sensing outcome. Specifically, in the $k$ th slot, if the SU senses a busy channel $q=k \bmod N$, no transmission is made. Otherwise it will transmit in the sensed channel $q$ with probability $\beta_{q}^{\mathrm{MA}}$.

To obtain the expression of $\beta_{q}^{\mathrm{MA}}$, we impose the collision constraint on channel $q$, i.e., the conditional probability of collision is bounded by $\gamma_{q}$. Note that the probability of the $\mathrm{SU}$ transmitting on channel $q$ is given by $v_{q}(0) \beta_{q}^{\mathrm{MA}} / N$ (the SU visit channel $q$ once in $N$ slots). The probability that, given the channel $q$ is idle at the beginning of slot $k, \mathrm{PU}$ starts to transmit in slot $k$ is $1-\exp \left(-\lambda_{q} T\right)$. Thus the product of $\left(1-\exp \left(-\lambda_{q} T\right)\right)$ and $v_{q}(0) \beta_{q}^{\mathrm{MA}} / N$ gives the probability SU and PU both transmits in channel $q$. Note further that (unconditional) the probability that the PU transmits in slot $k$ is $\left(1-v_{q}(0) \exp \left(-\lambda_{q} T\right)\right)$. Therefore, to satisfy the collision constraint $\gamma_{q}$, the transmission probability on channel $q \beta_{q}^{\mathrm{MA}}$ must be such that

$$
\frac{1}{N} v_{q}(0) \beta_{q}^{\mathrm{MA}} \frac{1-e^{-\lambda_{q} T}}{1-v_{q}(0) e^{-\lambda_{q} T}} \leq \gamma_{q} .
$$

Including the trivial condition $\beta_{q}^{\mathrm{MA}} \leq 1$, we have ${ }^{1}$

$$
\begin{aligned}
\beta_{q}^{\mathrm{MA}} & =\min \left(\frac{\gamma_{q} N\left(1-v_{q}(0) \exp \left(-\lambda_{q} T\right)\right)}{v_{q}(0)\left(1-\exp \left(-\lambda_{q} T\right)\right)}, 1\right) \\
& =\min \left(\frac{\gamma_{q} N \phi_{q}}{v_{q}(0)}, 1\right),
\end{aligned}
$$

where, for notational convenience, we denote

$$
\phi_{q} \triangleq \frac{1-v_{q}(0) \exp \left(-\lambda_{q} T\right)}{1-\exp \left(-\lambda_{q} T\right)}, \quad q=0, \cdots, N-1 .
$$

The throughput of this policy is then given by

$$
J\left(\pi^{\mathrm{MA}}\right)=\frac{1}{N} \sum_{i=0}^{N-1} v_{i}(0) \beta_{i}^{\mathrm{MA}} \exp \left(-\lambda_{i} T\right),
$$

where $v_{i}(0) \beta_{i}^{\mathrm{MA}}$ is the probability that the channel $i$ is idle and the SU transmits and $\exp \left(-\lambda_{i} T\right)$ is the probability that channel remains idle. The term inside the summation therefore is the probability of successful transmission by the SU on channel $i$, or the average number of packets successfully received.

\section{Optimality of Periodic Sensing and Memoryless Access}

We establish in this section that, under tight collision constraints, $J\left(\pi^{\mathrm{FO}}\right)=J\left(\pi^{\mathrm{MA}}\right)$, which necessarily means that $J\left(\pi^{*}\right)=J\left(\pi^{\mathrm{MA}}\right)$, i.e. PS-MA is optimal. We separate the heterogeneous case when PU traffic and constraints are different in each channel from the homogeneous counterpart. The latter greatly simplifies the derivation and provides insights into the main result of this paper.

\section{A. Intuitions: analysis for the homogeneous networks}

For homogeneous networks, traffic statistics in all channels are identical $\left(\lambda_{i}=\lambda, v_{i}(0)=v(0)\right)$ and their constraints the same $\left(\gamma_{i}=\gamma\right)$. The PS-MA then has the same transmission probability $\beta_{i}^{\mathrm{MA}}=\beta^{\mathrm{MA}}$. In this case, the performance is easily established. In particular, the throughput of PS-MA is given by the probability of SU's successful transmission, which is the product of $\beta^{\mathrm{MA}}$ and the probability that PU does not transmit, i.e.,

$$
J\left(\pi^{\mathrm{MA}}\right)=v(0) e^{-\lambda T} \beta^{\mathrm{MA}} .
$$

The following proposition, easily obtained by substituting $\beta^{\mathrm{MA}}$ from (9-10) into the above, highlights the relation between throughput and collision constraint $\gamma$.

Proposition 1: Given a homogeneous network of $N$ Markovian channels with traffic parameters $(\lambda, \mu)$ and stationary distribution $v(0)$ for the channel being idle, let

$$
\phi \equiv \frac{1-v(0) \exp (-\lambda T)}{1-\exp (-\lambda T)}, \quad \gamma^{\mathrm{MA}} \equiv \frac{v(0)}{N \phi} .
$$

The throughput of the PS-MA performance is

$$
J\left(\pi^{\mathrm{MA}}\right)=\left\{\begin{array}{cc}
N \phi \exp (-\lambda T) \gamma, & \gamma \in\left[0, \gamma^{\mathrm{MA}}\right] \\
\nu(0) \exp (-\lambda T), & \gamma \in\left(\gamma^{\mathrm{MA}}, 1\right] .
\end{array}\right.
$$

Note first that when the collision constraint is loose, the SU will always transmit whenever the channel is sensed idle. Therefore, the throughput is merely the probability that a

\footnotetext{
${ }^{1}$ We note that $\beta_{q}^{\mathrm{MA}}$ here is slightly different from the policy showed in the previous work [11] by adding $v_{q}(0)$ in the denominator in (9).
} 
channel is idle and remain idle for the duration $T$, which is the second part of (14). The breaking point $\gamma^{\mathrm{MA}}$ therefore is obtained when $\beta^{\mathrm{MA}}=1$. From (10), we have (13). The first part of (14) corresponds to the case when $\beta^{\mathrm{MA}}<1$. It is apparent that the throughput is proportional to $\beta^{\mathrm{MA}}$ and, from (10), proportional to the collision constraint $\gamma$.

For homogeneous networks, the analysis of FO-OSA can be obtained similarly. First, if the constraint is loose, then FO-OSA will always transmit, as long as there exists at least one channel that is idle. Therefore the probability of successful transmission (also the throughput) is given by $\left(1-v(1)^{N}\right) \exp (-\lambda T)$

When the collision constraint is tighter, FO-OSA will have to transmit probabilistically on some channel that is sensed idle. Since all the channels are statistically identical, the probability of the SU transmitting on any channel is the same, say $\beta^{\mathrm{FO}}$. Under the collision constraint $\gamma, \beta^{\mathrm{FO}}$ must satisfy

$$
\frac{1}{N}\left(1-v(1)^{N}\right) \beta^{\mathrm{FO}} \frac{1-e^{-\lambda T}}{1-v(0) e^{-\lambda T}} \leq \gamma .
$$

Note that the above condition is the same as (8) for PS-MA except that the probability of the specific sensed channel being idle is replaced by the probability that at least one channel is idle. Therefore, we have

$$
\beta^{\mathrm{FO}}=\min \left(\frac{\gamma N \phi}{\left(1-v(1)^{N}\right)}, 1\right),
$$

where $\phi$ is the same as that defined in (13). Setting $\beta^{\mathrm{FO}}=1$, we obtain the threshold $\gamma^{\mathrm{FO}}$ on constraint $\gamma$ above which FOOSA always transmits. See (15). The throughput of FO-OSA is then given by

$$
J\left(\pi^{\mathrm{FO}}\right)=\left(1-v(1)^{N}\right) e^{-\lambda T} \beta^{\mathrm{FO}} .
$$

Again, to highlight the relation between $J\left(\pi^{\mathrm{FO}}\right)$ and the collision constraint $\gamma$, we have the following proposition:

Proposition 2: Given a homogeneous network of $N$ Markovian channels with traffic parameters $(\lambda, \mu)$ and stationary distribution $v(0)$ for the channel being idle, let

$$
\phi \equiv \frac{1-v(0) \exp (-\lambda T)}{1-\exp (-\lambda T)}, \quad \gamma^{\mathrm{FO}} \equiv \frac{1-v(1)^{N}}{N \phi} .
$$

The throughput of the FO-OSA problem is

$$
J\left(\pi^{\mathrm{FO}}\right)=\left\{\begin{array}{cc}
N \phi \exp (-\lambda T) \gamma, & \gamma \in\left[0, \gamma^{\mathrm{FO}}\right], \\
\left(1-v(1)^{N}\right) \exp (-\lambda T), & \gamma \in\left(\gamma^{\mathrm{FO}}, 1\right] .
\end{array}\right.
$$

Combining the above two propositions, we obtain the characterization of the performance gap between the lower and upper bounds on the optimal access policy:

$$
J\left(\pi^{\mathrm{FO}}\right)-J\left(\pi^{\mathrm{MA}}\right)= \begin{cases}0, & \gamma \in\left(0, \gamma^{\mathrm{MA}}\right], \\ \left(\gamma-\gamma^{\mathrm{MA}}\right) N \phi \exp (-\lambda T), & \gamma \in\left(\gamma^{\mathrm{MA}}, \gamma^{\mathrm{FO}}\right], \\ \left(v(1)-v(1)^{N}\right) \exp (-\lambda T), & \gamma \in\left(\gamma^{\mathrm{FO}}, 1\right] .\end{cases}
$$

We note that when the constraints are tight $\gamma \in\left(0, \gamma^{\mathrm{MA}}\right]$, we can apply the simpler policy PS-MA which performs the same as the optimal policy FO-OSA. When the constraints are loose, $\gamma \in\left(\gamma^{\mathrm{MA}}, 1\right]$, (17) states that the performance of PS-MA deviates that of FO-OSA.

\section{B. General results: performance in heterogeneous networks}

We now present general results for heterogeneous networks where channels have different traffic statistics and collision constraints. Specifically, the on-off traffic on the $i$ th channel is given by the holding times $\lambda_{i}^{-1}$ and $\mu_{i}^{-1}$ for idle and busy states, respectively. The (conditional) collision probability on channel $i$ is upper bounded by $\gamma_{i}$.

Again, we first characterize the performance for the PS-MA policy in the following proposition. The generalization from the homogeneous networks is trivial: PS-MA transmits on channel $i$ with probability $\beta_{i}^{\mathrm{MA}}$, and the calculation of $\beta_{i}^{\mathrm{MA}}$ is based on the collision constraint $\gamma_{i}$ and channel characteristics $\left(\lambda_{i}, \mu_{i}\right)$, according to (10). The overall throughput is given by

$$
J\left(\pi^{\mathrm{MA}}\right)=\frac{1}{N} \sum_{i=0}^{N-1} v_{i}(0) e^{-\lambda_{i} T} \beta_{i}^{\mathrm{MA}},
$$

which can be rewritten as a function of collision constraints $\gamma_{i}$ in the following proposition.

Proposition 3: Given $N$ independent Markov channels with parameters $\left(\lambda_{i}, \mu_{i}\right)$ and stationary distributions $v_{i}(0)$ for idle states. Let

$\phi_{i} \equiv \frac{1-v_{i}(0) \exp \left(-\lambda_{i} T\right)}{1-\exp \left(-\lambda_{i} T\right)}, \quad W_{i} \equiv \phi_{i} \exp \left(-\lambda_{i} T\right), \quad \gamma_{i}^{\mathrm{MA}} \equiv \frac{v_{i}(0)}{N \phi_{i}}$.

The throughput of PS-MA is given by

$$
J\left(\pi^{\mathrm{MA}}\right)=\sum_{i=0}^{N-1} W_{i}\left(\gamma_{i}^{\mathrm{MA}} 1_{\left[\gamma_{i}>\gamma_{i}^{\mathrm{MA}}\right]}+\gamma_{i} 1_{\left[\gamma_{i} \leq \gamma_{i}^{\mathrm{MA}}\right]}\right),
$$

where $1_{[\cdot]}$ is the indicator function.

The analysis of FO-OSA for the heterogeneous network is more tedious and complicated. Appendix B gives an algebraic proof of Proposition 4 below. In this case, one can no longer use a single transmission probability for each channel as in the case of homogeneous networks. If channel $i$ is sensed idle, whether it is selected for transmission depends on which other channels are also idle and their channel characteristics, and the occurrence of each scenario is dictated by the stationary distribution $f(x)$. But the key is that, once channel $i$ is selected for transmission, the contribution to collision is the same as that for the PS-MA case. Thus it is not surprising that, under tight collision constraints, the throughput will be in the form of

$$
J\left(\pi^{\mathrm{FO}}\right)=\sum_{i=0}^{N-1} v_{i}(0) e^{-\lambda_{i} T} \bar{\beta}_{i}=\sum_{i=0}^{N-1} W_{i} \gamma_{i},
$$

where $\bar{\beta}_{i}$ is the probability of transmission on channel $i$ (a weighted sum of transmission probabilities under different sensing outcomes) and $W_{i}$ is the same as that for the PS-MA case.

The following proposition formalize the above arguments.

Proposition 4: Given $N$ independent Markov channels with parameters $\left(\lambda_{i}, \mu_{i}\right)$ and stationary distributions $v_{i}(0)$ for idle states, let

$$
\phi_{i} \equiv \frac{1-v_{i}(0) \exp \left(-\lambda_{i} T\right)}{1-\exp \left(-\lambda_{i} T\right)}, \quad W_{i} \equiv \phi_{i} \exp \left(-\lambda_{i} T\right) .
$$


Let $f(x), x \in\{0,1\}^{N}$, be the stationary distribution of the $\mathrm{N}$ channel Markov process. Denote, for $k=1, \cdots, N$,

$$
\begin{aligned}
S_{i}^{k} & \equiv\left\{x \mid x \in\{0,1\}^{N}, x_{i}=0, x \text { contains total } k \text { zeros }\right\} \\
F_{i}^{k} & \equiv \sum_{x \in S_{i}^{k}} f(x), \quad \gamma_{i}^{\mathrm{FO}} \equiv \frac{1}{\phi_{i}} \sum_{j=1}^{N} \frac{F_{i}^{j}}{j} .
\end{aligned}
$$

If $\gamma_{i} \in\left[0, \gamma_{i}^{\mathrm{FO}}\right], \forall i$, then the throughput of the optimal FO-OSA policy can be given by

$$
J\left(\pi^{\mathrm{FO}}\right)=\sum_{i=0}^{N-1} W_{i} \gamma_{i}
$$

Otherwise, the throughput of the optimal FO-OSA policy is upper bounded by

$$
J\left(\pi^{\mathrm{FO}}\right) \leq \min \left(U, \sum_{i=0}^{N-1} W_{i} \gamma_{i}\right)
$$

where

$$
U \equiv v_{(0)}(0) \exp \left(-\lambda_{(0)} T\right)+\sum_{i=1}^{N-1}\left(\prod_{j=0}^{i-1} v_{(j)}(1)\right) v_{(i)}(0) \exp \left(-\lambda_{(i)} T\right),
$$

where we sort the channel in the decreasing order according to $\exp \left(-\lambda_{i} T\right)$, and the parameters with subscript " $(i)$ " means the sorted channel number.

Proof: See Appendix B.

We note from (18) and (21),

$$
\gamma_{i}^{\mathrm{FO}}=\frac{N \sum_{j=1}^{N}\left(F_{i}^{j} / j\right)}{N \phi_{i}} \geq \frac{\sum_{j=1}^{N} F_{i}^{j}}{N \phi_{i}}=\frac{v_{i}(0)}{N \phi_{i}}=\gamma_{i}^{\mathrm{MA}} \forall i .
$$

Therefore from Proposition 3 and Proposition 4, we obtain the following theorem:

Theorem 1: Given $N$ independent Markov channels with parameters $\left(\lambda_{i}, \mu_{i}\right)$ and stationary distributions $v_{i}(0)$ for idle states, let $\gamma_{i}^{\mathrm{MA}}$ be the threshold defined in (18) and $\gamma_{i}^{\mathrm{FO}}$ in (21). The performance gap between policy $\pi^{\mathrm{FO}}$ and $\pi^{\mathrm{MA}}$ is given by

$J\left(\pi^{\mathrm{FO}}\right)-J\left(\pi^{\mathrm{MA}}\right)= \begin{cases}0, & \gamma_{i} \in\left[0, \gamma_{i}^{\mathrm{MA}}\right], \forall i \\ \sum_{i=0}^{N-1} W_{i}\left(\gamma_{i}-\gamma_{i}^{\mathrm{MA}}\right) 1_{\left[\gamma_{i}>\gamma_{i}^{\mathrm{MA}}\right]}, & \gamma_{i} \in\left[0, \gamma_{i}^{\mathrm{FO}}\right], \forall i .\end{cases}$

In general,

$$
J\left(\pi^{\mathrm{FO}}\right)-J\left(\pi^{\mathrm{MA}}\right) \leq \min (\Delta U, \Delta W),
$$

where

$$
\begin{aligned}
\Delta U & =U-\sum_{i=0}^{N-1} W_{i}\left(\gamma_{i}^{\mathrm{MA}} 1_{\left[\gamma_{i}>\gamma_{i}^{\mathrm{MA}}\right]}+\gamma_{i} 1_{\left[\gamma_{i} \leq \gamma_{i}^{\mathrm{MA}}\right]}\right) \\
\Delta W & \equiv \sum_{i=0}^{N-1} W_{i}\left(\gamma_{i}-\gamma_{i}^{\mathrm{MA}}\right) 1_{\left[\gamma_{i}>\gamma_{i}^{\mathrm{MA}}\right]}
\end{aligned}
$$

where

$$
U \equiv v_{(0)}(0) e^{-\lambda_{(0)} T}+\sum_{i=1}^{N-1}\left(\prod_{j=0}^{i-1} v_{(j)}(1)\right) v_{(i)}(0) e^{-\lambda_{(i)} T} .
$$

Theorem 1 shows that when each channel collision constraint lies in the special interval, $\gamma_{i} \in\left[0, \gamma_{i}^{\mathrm{MA}}\right], \forall i$, the policy PS-MA also performs the same to the policy FO-OSA. Thus we conclude that, for heterogeneous networks, PS-MA is optimal under tight collision constraints.

\section{Extensions to Multi-User Access}

The extension to multiple SUs is challenging in general. The optimality of PS-MA under tight collision constraints, however, gives us some hope because its structure lends itself to a natural extension to the multiuser setting. Here we present some simple extensions to the multiuser case based applications of PS-MA. See [20] for general results.

\section{A. K-user Orthogonal PS-MA: $\pi_{K}^{\mathrm{MA}-\mathrm{O}}$}

Suppose that there is a way for each user to choose the sensing phase orthogonal to others. This can be easily accomplished if there is a base station that announces the total number of SUs and the sensing phase available to new SUs. Then transmissions of the SUs are completely orthogonalized.

The orthogonalized PS-MA, denoted as $\pi_{K}^{\mathrm{MA}-\mathrm{O}}$, operates as follows. Each SU senses all channels periodically with its own sensing phase. When the channel is busy, it goes to the next channel in the next slot. Otherwise, it transmits with a probability adjusted to accommodate the presence of other SUs.

To derive the transmission probabilities, we follow the single user case, except that each channel now has interference contributed not by one but $K$ SUs. To satisfy the collision constraint $\gamma_{i}$ for the $i$ th channel, we have

$$
\frac{K}{N} v_{i}(0) \beta_{i}^{\mathrm{MA}-\mathrm{O}} \frac{1-e^{-\lambda_{i} T}}{1-v_{i}(0) e^{-\lambda_{i} T}} \leq \gamma_{i} .
$$

Thus, in an $N$ channel system with $K \leq N$ SUs network with channel collision constraints $\gamma_{i}$, the SU that transmits in channel $i$ should scale the transmission probability for the single user case as

$$
\beta_{i, K}^{\mathrm{MA}-\mathrm{O}}=\min \left\{\frac{\gamma_{i} N \phi_{i}}{K v_{i}(0)}, 1\right\}
$$

Then the total throughput of the $K$ secondary users' access is

$$
J\left(\pi_{K}^{\mathrm{MA}-\mathrm{O}}\right)=\frac{K}{N} \sum_{i=0}^{N-1} v_{i}(0) \exp \left(-\lambda_{i} T\right) \beta_{i, K}^{\mathrm{MA}-\mathrm{O}} .
$$

Again we can evaluate the optimal FO-OSA strategy for $K$ SUs. Denoted as $\pi_{K}^{\mathrm{FO}}$, the $k$-user FO-OSA scheme allocate SUs on idle channels upon full spectrum sensing. If there are more SUs than idle channels, we randomly select, with equal probability, a subset of SUs to transmit probabilistically on all available idle channels. If there are less number of SUs than idle channels, we assign SUs randomly on a subset of channels and they transmit probabilistically. The probabilistic assignment needs to be optimized based on channel traffic statistics and collision constraints.

The analysis of this scheme is more complicated and we will not present our result here, except stating the simple case for $K=N$. In this case, there are always no less number of SUs than the available idle channels. It is clear that the orthogonal PS-MA is identical to the $K$-user FO-OSA. 


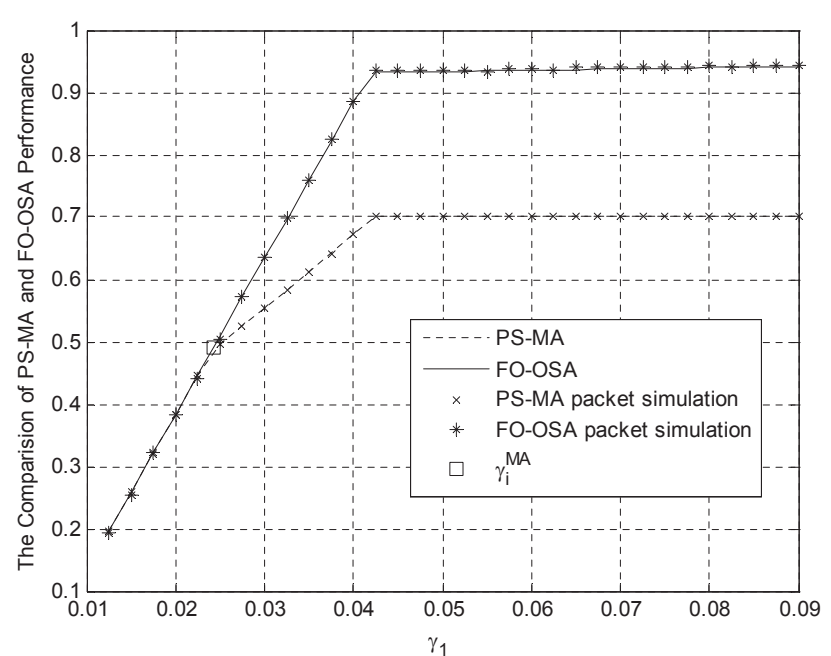

Fig. 2. The comparison of PS-MA and FO-OSA throughputs of the SU with $N=6$. The parameters are setting as $N=6: \lambda_{0}^{-1}=\lambda_{2}^{-1}=\lambda_{4}^{-1}=$ $4.20 \mathrm{~ms}, \lambda_{1}^{-1}=\lambda_{3}^{-1}=\lambda_{5}^{-1}=3.23 \mathrm{~ms}, \mu_{0}^{-1}=\mu_{2}^{-1}=\mu_{4}^{-1}=1.00 \mathrm{~ms}, \mu_{1}^{-1}=$ $\mu_{3}^{-1}=\mu_{5}^{-1}=1.43 \mathrm{~ms}$ and $\gamma_{0}=\gamma_{2}=\gamma_{4}, \gamma_{1}=\gamma_{3}=\gamma_{5}=\gamma_{0}+0.01$;

\section{B. K-user random access}

The orthogonal random access has well known shortcomings: the need of a central controller and the number of SUs must be less than $N$. Given that SUs may only need the channel sporadically, it is natural to consider random access schemes.

For simplicity, we present a CSMA-like random access extension of PS-MA. Each SU randomly chooses, with equal probability, a channel to sense. If the channel is busy, it will not transmit. Otherwise, it will continue to sense until a random backoff time. If no one transmits at the end of the backoff time, it will transmit with certain probability computed to ensure the collision constraint on that channel be satisfied.

The access probability is given by

$$
\beta_{i, K}^{\mathrm{MA}-\mathrm{CSMA}}=\min \left\{N\left(1-\left(1-\frac{\gamma_{i} \phi_{i}}{v_{i}(0)}\right)^{\frac{1}{K}}\right), 1\right\} .
$$

If we ignore the delay of carrier sensing detection, then the total throughput of the $K$ secondary users' access is (32), where $P_{K}(j)=C(K, j)\left(\frac{1}{N}\right)^{j}\left(\frac{N-1}{N}\right)^{K-j}$ means the probability that one particular channel can be selected by $0 \leq j \leq K$ SUs simultaneously when there are $N$ channels. It can be seen that asymptotically, it achieves the FO-OSA performance as the number of users increases.

\section{Numerical Simulation}

We now present some numerical results to illustrate the performance using specific network parameters. The selections of these parameters are not crucial, and our simulation results are representative of the general behavior of the network. Specifically, we assume the slot size $T=0.25 \mathrm{~ms}$. Specific parameters are listed in the figure captions.

\section{A. Performance of Single User Policies}

We performed the packet-level Monte-Carlo simulations and compared the results with the analytical expressions presented

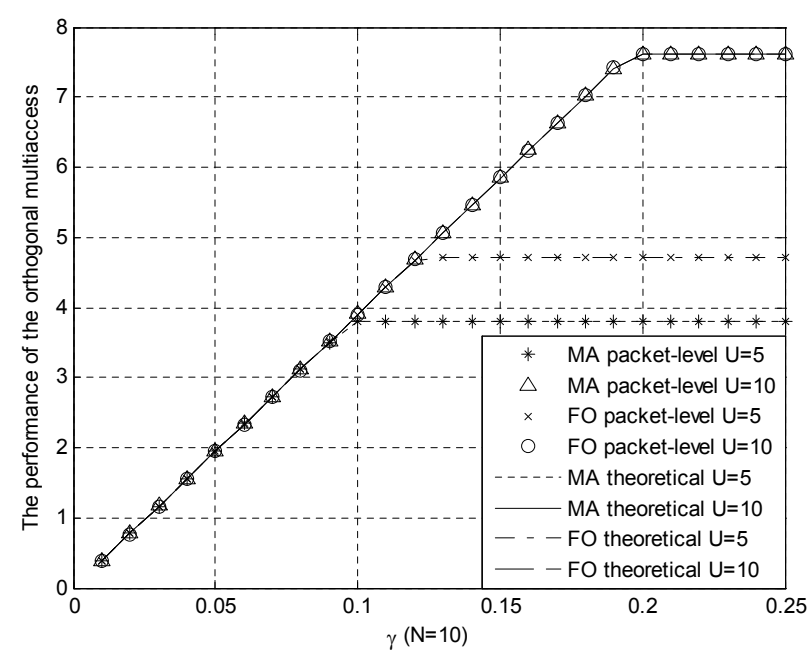

Fig. 3. The performances of the random access orthogonalized PS-MA and FO-OSA policies in homogeneous networks. The parameters are setting as $N=10, U=5,10 ; \lambda^{-1}=4.20 \mathrm{~ms}, \mu^{-1}=1.00 \mathrm{~ms}$.

in Proposition 3 and Proposition 4. The running time length of the packet-level simulation was set as $10000 \mathrm{~ms}$. We also performed the comparison of the performances of PS-MA and FO-OSA to validate the result of Theorem 1. In this section, we focus on the case $N=6$, and consider the trend of throughput variation as we loosen the constraints.

Fig. 2 shows the results from packet level simulation and the analytical expressions for the $N=6$ channel case. It is evident that the packet level simulation matches the analytical expressions. It also shows that, when the collision constraints are tight, FO-OSA and PS-MA match. When the collision constraint is loose, both policies saturate. For PS-MA, it means that all the constraints satisfy $\gamma_{i} \in\left(\gamma_{i}^{\mathrm{MA}}, 1\right], \forall i$. For FO-OSA, it means no collision constraints. The computed collision threshold is given by $\gamma_{1}^{\mathrm{MA}}(N=6)=0.024$, which also matches with the simulation result.

\section{B. Performance of Multi-User Policies}

We also performed the packet-level Monte-Carlo simulations for multi-user access policies. The running time length of the simulation was also set as $10000 \mathrm{~ms}$.

In the simulation, we considered $N=10$ channels. In Fig 3 , we compared the orthogonalized multi-user access policies PS-MA and FO-OSA when the SU's number is $U=5$ and $U=$ 10 respectively. Again, the packet-level simulations matched to the analytical results in both the two policies. For the case involving $U=10$ secondary users, we observed that the PSMA and FO-OSA performed the same as discussed in Section $\mathrm{V}-\mathrm{A}$.

In Fig 4, we gave the performances of the random access policy CSMA in different SU's nmuber $U=5, U=10, U=$ 20 and $U=50$ when the channel number is also $N=10$. Besides showing the match between the simulation results and the analytical results of CSMA, it also showed that when the SU's number increased, the maximum achievable throughput of the CSMA policy approached to that of the orthogonalized FO-OSA policy of the case $U=10$. 


$$
J\left(\pi_{i, K}^{\mathrm{MA}-\mathrm{CSMA}}\right)=\sum_{i=0}^{N-1} \sum_{j=1}^{K} P_{K}(j)\left(1-\left(1-\beta_{i, K}^{\mathrm{MA}-\mathrm{CSMA}}\right)^{j}\right) v_{i}(0) \exp \left(-\lambda_{i} T\right)
$$

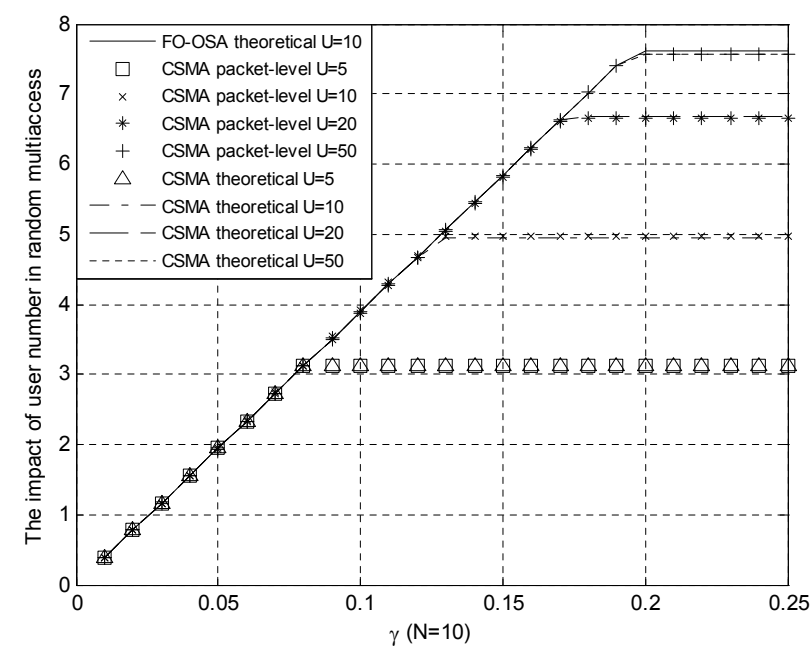

Fig. 4. The performances of random access policies CSMA. The parameters are setting as $N=10, U=5,10,20,50 ; \lambda^{-1}=4.20 \mathrm{~ms}, \mu^{-1}=1.00 \mathrm{~ms}$.

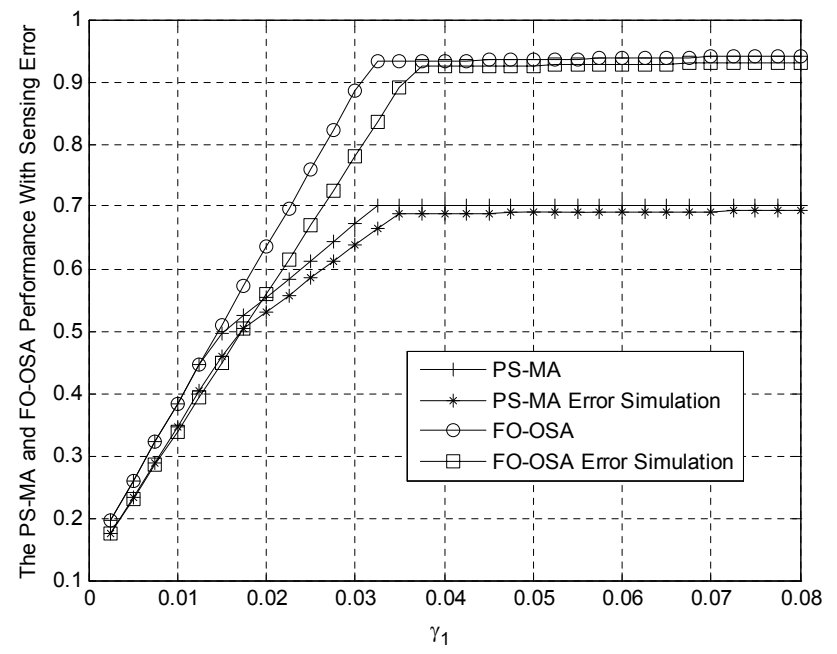

Fig. 5. The performances of optimal PS-MA and FO-OSA policies when there exists sensing error. The parameters are setting as $N=10, U=$ 5,$10 ; \lambda^{-1}=4.20 \mathrm{~ms}, \mu^{-1}=1.00 \mathrm{~ms}$.

We also examined the case when sensing is not perfect. In particular, we assumed that the probabilities of detection (both false alarm and miss detection) were 0.02. Given these detection error probabilities, transmission probabilities were adjusted accordingly to satisfy the collision constraints. The simulation results are shown in Fig. 5 where we see that the PS-MA and FO-OSA have the performance degradation less than $1.8 \%$ and $1.1 \%$ respectively comparing with the perfect sensing case. We also note that, in the presence of sensing noise, the PS-MA performed the same as the FO-OSA when the constraints were tight. Fig. 6 shows that the collision rates in both PS-MA and FO-OSA cases satisfied the collision constraints.

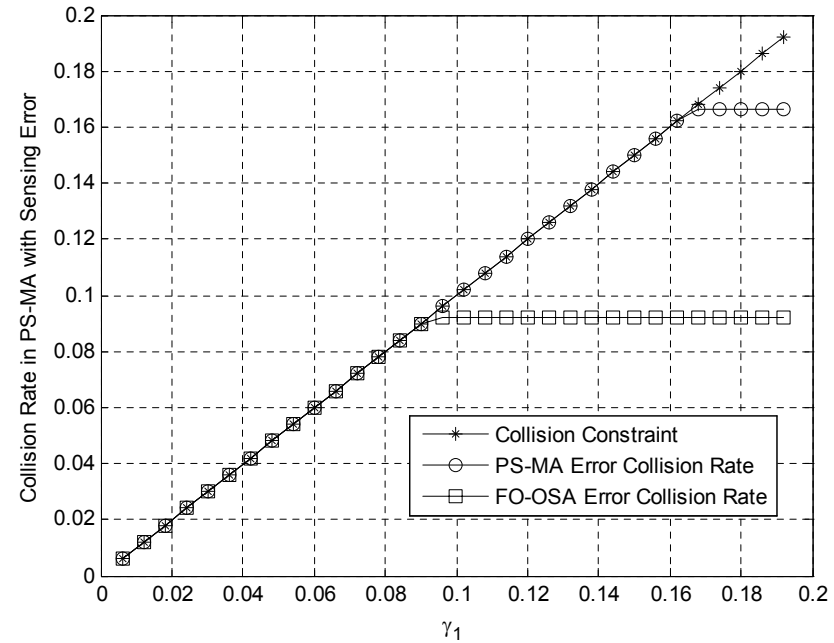

Fig. 6. The collision rate of optimal PS-MA and FO-OSA policies when there exists sensing error. The parameters are setting as $N=10, U=$ $5,10,20,50 ; \lambda^{-1}=4.20 \mathrm{~ms}, \mu^{-1}=1.00 \mathrm{~ms}$.

\section{Conclusion}

The main result presented in this paper is that, under tight collision constraints, optimal cognitive access of multiple continuous-time on-off Markovian channels can be achieved by deterministic periodic sensing and randomized transmission policies. This result has several important theoretical and practical ramifications. From a theoretical view point, characterizing optimal sensing and access policy is in general difficult and is still an open problem. Our result, though only limitted to access with tight collision constraints, is the first solution to this problem. Furthermore, the structure of PSMA also makes it possible to extend the result to the multiuser setting. More significant of this result is perhaps in its practical aspects. The implementation of PS-MA is quite simple, and it can be used for distributed access of multiple secondary users.

There are several weaknesses associated with the proposed model and analysis, some of these are difficult to address analytically. The continuous-time Markov chain (CTMC) model of channel occupancy is an idealization that can only be an approximation to practical situations. For example, the CTMC can switch arbitrary number of times for any finite time duration. For practical bandlimitted channel, this would not be possible. The synchronization of secondary users is a significant restriction. In practice, unless the SUs have their own basestation, synchronization among distributed secondary users seems to be extremely difficult. Sensing error is another practical issue that needs to be addressed. In this paper, we have included some numerical robustness simulations. While the results seem to suggest that the performance degrades gracefully when there is moderate sensing errors, it is desirable to quantify such degradations. All these issues are worthy of further study. 


\section{Appendix A}

\section{The Proof of Proposition 1}

Proof: In homogeneous networks, from the MA optimal policy structure (10), we get the $\gamma^{\mathrm{MA}}$ by letting $\frac{\gamma N \phi}{v(0)}=1$, and the policy is thus:

$$
\beta^{\mathrm{MA}}=\left\{\begin{array}{cl}
\frac{\gamma N \phi}{v(0)}, & \gamma \in\left[0, \gamma^{\mathrm{MA}}\right] \\
1, & \gamma \in\left(\gamma^{\mathrm{MA}}, 1\right] .
\end{array}\right.
$$

Therefore, the throughput expression can be obtained from (12) as

$$
J\left(\pi^{\mathrm{MA}}\right)= \begin{cases}N \phi \exp (-\lambda T) \gamma, & \gamma \in\left[0, \gamma^{\mathrm{MA}}\right] \\ v(0) \exp (-\lambda T), & \gamma \in\left(\gamma^{\mathrm{MA}}, 1\right]\end{cases}
$$

\section{Appendix B}

The Proof of Proposition 4

Proof: We consider the following linear programming problem which has the equivalent throughput of the original FO-OSA problem:

$$
\max _{\beta} \sum_{x \in\{0,1\}^{N}} \sum_{i=0}^{N-1} f(x) 1_{\left(x_{i}=0\right)} \cdot \beta_{i}(x) \exp \left(-\lambda_{i} T\right)
$$

subject to

$$
\begin{gathered}
\sum_{x \in\{0,1\}^{N}} \frac{f(x) 1_{\left(x_{i}=0\right)} \cdot \beta_{i}(x)}{\phi_{i}} \leq \gamma_{i}, \quad \forall i \\
\sum_{i=0}^{N-1} \beta_{i}(x) \leq 1, \forall x ; \quad \beta_{i}(x) \in[0,1], \forall x, i .
\end{gathered}
$$

Recall that

$$
\phi_{i}=\frac{\left(1-v_{i}(0) \exp \left(-\lambda_{i} T\right)\right)}{1-\exp \left(-\lambda_{i} T\right)}
$$

and

$$
W_{i}=\exp \left(-\lambda_{i} T\right) \phi_{i}
$$

Let $B^{\mathrm{FO}}$ be the set of all the feasible solutions of this optimization problem and an optimal policy be $\pi^{\mathrm{FO}}$. Multiply both sides of each inequalities in (36) with $W_{i}$ respectively, and sum up all the $N$ inequalities and we can get the following inequality for all $\beta \in B^{\mathrm{FO}}$ :

$$
\sum_{x \in\{0,1\}^{N}} \sum_{i=0}^{N-1} f(x) 1_{\left(x_{i}=0\right)} \cdot \beta_{i}(x) \exp \left(-\lambda_{i} T\right) \leq \sum_{i=0}^{N-1} W_{i} \gamma_{i} .
$$

This provides an upper bound of the optimal objective (35).

At the same time, we consider a different upper bound of the FO-OSA throughput (35) by ignoring the collision constrains by letting $\gamma_{i}=1, \forall i$. For heterogeneous networks, the transmission rule is we give the priority to transmit in the most likely idle channel. In FO-OSA, it means that if the sensing result shows that a few channels are idle, we will transmit in the channel that can give the biggest reward. Then this upper bound can be expressed as

$$
U \equiv v_{(0)}(0) \exp \left(-\lambda_{(0)} T\right)+\sum_{i=1}^{N-1}\left(\prod_{j=0}^{i-1} v_{(j)}(1)\right) v_{(i)}(0) \exp \left(-\lambda_{(i)} T\right) \text {. }
$$

Here we sort the channels in the decreasing order of $\exp \left(-\lambda_{i} T\right)$, and the parameters with subscript " $(i)$ " means the sorted channel number.
Therefore we can first conclude that (23) is true.

It remains to show that under tight constraints $\left(\gamma_{i} \in\right.$ $\left.\left(0, \gamma_{i}^{\mathrm{FO}}\right], \forall i\right)$, this upper bound $\sum_{i} W_{i} \gamma_{i}$ becomes exact. Define an increasing sequence $\gamma_{i}^{(k)}$ as

$$
\gamma_{i}^{(k)}=\left\{\begin{array}{cc}
0, & k=0 \\
\frac{\sum_{j=1}^{k}\left(F_{i}^{j} / j\right)}{\phi_{i}}, & k=1,2, \ldots, N,
\end{array}\right.
$$

where $\gamma_{i}^{(N)}=\gamma_{i}^{\mathrm{FO}}$.

We define the non-zero elements of our policy $\beta$ depending on the position of $\gamma_{i}$ in the interval $\left(0, \gamma_{i}^{\mathrm{FO}}\right]$ as follows. Assume $\gamma_{i} \in\left[\gamma_{i}^{(k-1)}, \gamma_{i}^{(k)}\right], k=1,2, \ldots, N$,

$$
\beta_{i}(x) \equiv \begin{cases}\gamma_{i} \phi_{i} / F_{i}^{1}, & k=1 ; \\ 1 / j, & x \in S_{i}^{j}, j=1, \ldots, k-1, k>1 ; \\ \Delta \beta_{i}, & x \in S_{i}^{k},\end{cases}
$$

where

$$
\Delta \beta_{i}=\frac{\gamma_{i} \phi_{i}-\sum_{j=1}^{k-1}\left(F_{i}^{j} / j\right)}{F_{i}^{k}}, 1<k \leq N .
$$

Let us first verify that the policy $\beta$ satisfies the constraint (37). We divide the proof into 3 cases based on the position of $x$.

Case 1. When $x \in \cup_{i} S_{i}^{j}$ with $j=1,2, \cdots, k-1$, i.e., there are $j 0 \mathrm{~s}$ in $x, \beta_{i}(x)=\frac{1}{j} 1_{x_{i}=0}$. Thus

$$
\sum_{i} \beta_{i}(x)=\sum_{i}(1 / j) 1_{x_{i}=0}=1 .
$$

Case 2. When $x \in \cup_{i} S_{i}^{k}$, i.e., there are $k$ os in $x, \beta_{i}(x)=$ $\Delta \beta_{i} 1_{x_{i}=0}$. We can see that in (41), $\Delta \beta_{i}$ is an increasing function of $\gamma_{i}$. Thus when $\beta_{i}(x)=\Delta \beta_{i} \leq \Delta \beta_{i}\left(\gamma_{i}^{(k)}\right), x_{i}=0$,

$$
\begin{aligned}
\Delta \beta_{i}\left(\gamma_{i}^{(k)}\right) & =\frac{\gamma_{i}^{(k)} \phi_{i}-\sum_{j=1}^{k-1}\left(F_{i}^{j} / j\right)}{F_{i}^{k}} \\
& =\frac{\frac{\sum_{j=1}^{k}\left(F_{i}^{j} / j\right)}{\phi_{i}} \phi_{i}-\sum_{j=1}^{k-1}\left(F_{i}^{j} / j\right)}{F_{i}^{k}} \\
& =\left(F_{i}^{k} / k\right) / F_{i}^{k}=\frac{1}{k} .
\end{aligned}
$$

So

$$
\sum_{i} \beta_{i}(x) \leq k \Delta \beta_{i}\left(\gamma_{i}^{(k)}\right)=1 .
$$

Case 3. For other $x$, i.e., there are $j=k+1, k+2, \cdots, N$ os or no 0 in $x, \beta_{i}(x)=0$. Thus $\sum_{i} \beta_{i}(x)=0$.

Combining all three cases, we can conclude that the inequalities in (37) are always satisfied for all $x$.

Next we verify that for the policy $\beta$, all the $N$ equal marks in (36) are satisfied simultaneously.

Case 1. Consider the case $k=1$, i.e., $\gamma_{i} \in\left(\gamma_{i}^{(0)}=0, \gamma_{i}^{(1)}\right]$. For $x \in S_{i}^{1}$ (there is only one such $x$, thus $f(x)=F_{i}^{1}$ ), according to $(40), \beta_{i}(x)=\Delta \beta=\gamma_{i} \phi_{i} / F_{i}^{1}$ holds; For $x \in\{0,1\}^{N}-S_{i}^{1}$, $\beta_{i}(x)=0$. Thus the evaluation of the left hand side of the $i$-th inequality in (36) is given by

$$
\frac{f(x) \cdot\left(\gamma_{i} \phi_{i} / F_{i}^{1}\right)}{\phi_{i}}=\gamma_{i} .
$$

Thus the $i$-th inequality (36) becomes equality. 
Case 2. Consider the case $k>1$. Let $i$ be a given channel. For the case where $x \in S_{i}^{j}$ for some $j, 0<j<k$, we have $\beta_{i}(x)=1 / j$ according to (40). For $x \in S_{i}^{k}$, we have

$$
\beta_{i}(x)=\Delta \beta_{i}=\frac{\gamma_{i} \phi_{i}-\sum_{j=1}^{k-1}\left(F_{i}^{j} / j\right)}{F_{i}^{k}},
$$

according to (41). For all other $x, \beta_{i}(x)=0$. Thus the left hand side of the $i$-th inequality (36) can be evaluated under the policy $\beta$ defined by (40) as

$$
\left[\sum_{j=1}^{k-1} F_{i}^{j} \cdot \frac{1}{j}+F_{i}^{k} \cdot \frac{\gamma_{i} \phi_{i}-\sum_{j=1}^{k-1}\left(F_{i}^{j} / j\right)}{F_{i}^{k}}\right] / \phi_{i}=\gamma_{i} .
$$

We can see that the $i$-th inequality in (36) becomes equality.

Combining the two cases, we can see that as long as $\gamma_{i}$ is within the interval $\left[0, \gamma_{i}^{\mathrm{FO}}\right]$, the $i$-th inequality in (36) becomes equality under the policy $\beta$. Since $i$ is arbitrary, we can conclude that all inequalities in (36) become equalities under the policy $\beta$ when $\gamma_{i} \in\left[0, \gamma_{i}^{\mathrm{FO}}\right]$ holds for all $i$. Therefore the upper bound $\sum_{i=0}^{N-1} W_{i} \gamma_{i}$ introduced in (38) is achievable when $\gamma_{i} \in$ $\left[0, \gamma_{i}^{\mathrm{FO}}\right], \forall i$. That is when $\gamma_{i} \in\left(0, \gamma_{i}^{\mathrm{FO}}\right], \forall i=0,1, \cdots, N-1$,

$$
J\left(\pi^{\mathrm{FO}}\right)=\sum_{i=0}^{N-1} W_{i} \gamma_{i}
$$

\section{REFERENCES}

[1] Q. Zhao and B. M. Sadler, "A survey of dynamic spectrum access," IEEE Signal Process. Mag., vol. 24, pp. 79-89, May 2007.

[2] Q. Zhao, L. Tong, and A. Swami, "Decentralized Cognitive MAC for Dynamic Spectrum Access," in Proc. first IEEE Symposium on New Frontiers in Dynamic Spectrum Access Networks (DySPAN), (Baltimore, MD), Nov 2005.

[3] S. Geirhofer, L. Tong, and B. M. Sadler, "Cognitive medium access: constraining interference based on experimental models," IEEE J. Sel. Areas Commun., Special Issues on Cognitive Radio: Theory and Applications, vol. 36, pp. 785-796, Feb 2008.

[4] H. Yu, Approximation solution methods for partially observable Markov and semi-Markov decision processes. $\mathrm{PhD}$ thesis, Department of Electrical Engineering and Computer Science, MIT, Cambridge, MA, 2007.

[5] I. F. Akyildiz, W. Y. Lee, M. Vuran, and S. Mohanty, "A survey on spectrum Management in cognitive radio networks," IEEE Commun. Mag., April 2008.

[6] Q. Zhao, L. Tong, A. Swami, and Y. Chen, "Decentralized Cognitive MAC for Opportunistic Spectrum Access in Ad Hoc Networks: A POMDP Framework," IEEE J. Sel. Areas Commun., vol. 25, pp. 589600, April 2007.

[7] Y. Chen, Q. Zhao, and A. Swami, "Joint Design and Separation Principle for Opportunistic Spectrum Access in the Presence of Sensing Errors," IEEE Tran. Inf. Theory, vol. 54, pp. 2053-2071, May 2008.

[8] Q. Zhao, B. Krishnamachari, and K. Liu, "On Myopic Sensing for MultiChannel Opportunistic Access: Structure, Optimality, and Performance," IEEE Trans. Wireless Commun., vol. 7, pp. 5431-5440, Dec 2008.

[9] S. Ahmad, M. Liu, T. Javidi, Q. Zhao, and B. Krishnamachari, "Optimality of myopic sensing in multi-channel opportunistic access," IEEE Trans. Inf. Theory, vol. 55, no. 9, pp. 4040-4050, 2009.

[10] Q. Zhao, S. Geirhofer, L. Tong, and B. Sadler, "Optimal dynamic spectrum access via periodic channel sensing," in Proc. Wireless Communications and Networking Conference (WCNC), (Hong Kong), March 2007.

[11] Q. C. Zhao, S. Geirhofer, L. Tong, and B. M. Sadler, "Opportunistic Spectrum Access via Periodic Channel Sensing," IEEE Trans. Signal Process., vol. 36, pp. 785-796, Feb 2008.
[12] S. Geirhofer, L. Tong, and B. M. Sadler, "Dynamic Spectrum Access in the Time Domain: Modeling and Exploiting Whitespace," IEEE Commun. Mag., vol. 45, pp. 66-72, May 2007.

[13] S. Huang, X. Liu, and Z. Ding, "Opportunistic spectrum access in cognitive radio networks," in Proc. 2008 IEEE INFOCOM, (Phoenix, AZ), 2008.

[14] S. Geirhofer, L. Tong, and B. Sadler, "Interference-aware OFDMA resource allocation: A predictive approach," in Proc. 2008 IEEE Military Comm. Conf. (MILCOM), pp. 1-7, Oct. 2008.

[15] S. Huang, X. Liu, and Z. Ding, "Optimal sensing-transmission structure for dynamic spectrum access," in Proc. 2009 IEEE INFOCOM, 2009.

[16] I. A. Akbar and W. H. Tranter, "Dynamic spectrum allocation in cognitive radio using hidden Markov models: Poisson distributed case," in IEEE Proc. SoutheastCon, pp. 196-201, March 2007.

[17] S. Wang, J. Zhang, and L. Tong, "Delay analysis for cognitive radio networks with random access: A fluid queue view," in Proc. 2010 IEEE INFOCOM, (San Diego, CA), pp. 1-9, Apr 2010.

[18] K. Liu and Q. Zhao, "Decentralized multi-armed bandit with multiple distributed players," in Information Theory and Applications Workshop (ITA), pp. 1-10, Jan. 2010

[19] A. Anandkumar, N. Michael, and A. Tang, "Opportunistic Spectrum Access with Multiple Users: Learning under Competition," in Proc. of IEEE INFOCOM, (San Deigo, USA), Mar. 2010.

[20] S. Chen and L. Tong, "Multiuser cognitive access of continuous time markov channels: maximum throughput and effective bandwidth regions," in Proc. 2010 Information Theory and Applications Workshop, (San Diego, CA), Jan. 2010.

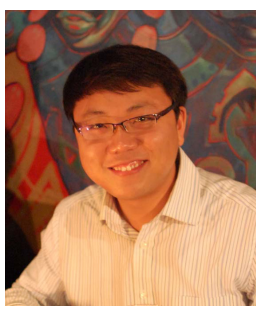

Xin Li (S'10) received the B.E. degree in communication engineering, from Harbin Institute of Technology, Harbin, China, in 2005. Since then he has been working toward the Ph.D. in the Center for Intelligent and Networked Systems (CFINS) in the Department of Automation at Tsinghua University, Beijing, China. His research interests focus on wireless sensor networks, dynamic spectrum access and cognitive radio networks.

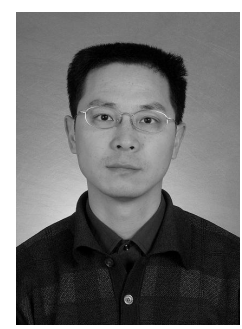

Qianchuan Zhao (M'06-SM'08) received the B.E. degree in automatic control in July 1992, the B.S degree in applied mathematics in July 1992, and $\mathrm{Ph} . \mathrm{D}$. degree in control theory and its applications in July 1996, all from Tsinghua University, Beijing, China.

$\mathrm{He}$ is currently a Professor and Associate Director of the Center for Intelligent and Networked Systems (CFINS), Department of Automation, Tsinghua University. He was a Visiting Scholar at Carnegie Mellon University, Pittsburgh, PA, and Harvard University, Cambridge, MA, in 2000 and 2002, respectively. He was a Visiting Professor at Cornell University, Ithaca, NY, in 2006. His research interests include modeling, analysis, control and optimization for complex networked systems. He is an associate editor for Journal of Optimization Theory and Applications and an associate editor for IEEE Transactions on Automation Science and Engineering. 


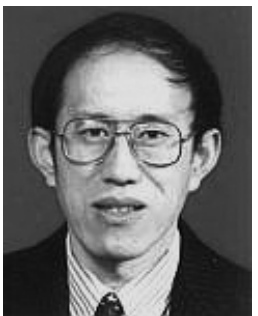

Xiaohong Guan (M'93-SM'95-F'07) received the B.S. and M.S. degrees in automatic control from Tsinghua University, Beijing, China, in 1982 and 1985, respectively, and the Ph.D. degree in electrical engineering from the University of Connecticut, Storrs, in 1993. He was a Senior Consulting Engineer with PG\&E from 1993 to 1995. From 1985 to 1988 , and since 1995 , he has been with the Systems Engineering Institute at Xian Jiaotong University, Xian, China, and currently, he is the Cheung Kong Professor of Systems Engineering, the Dean of School Electronic and Information Engineering and Director of Systems Engineering Institute. He is also the Director of Center for Intelligent and Networked Systems and served the Head of Department of Automation, Tsinghua University from 2003 to 2008. He visited the Division of Engineering and Applied Science, Harvard University from January 1999 to February 2000. His research interests include scheduling of power and manufacturing systems, bidding strategies for deregulated electric power markets, economy and security of complex network systems and sensor networks.

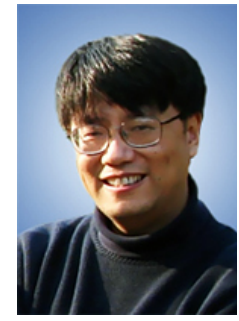

Lang Tong (S'87,M'91,SM'01,F'05) is the Irwin and Joan Jacobs Professor in Engineering at Cornell University Ithaca, New York. He received the B.E. degree from Tsinghua University, Beijing, China, in 1985, and M.S. and Ph.D. degrees in electrical engineering in 1987 and 1991, respectively, from the University of Notre Dame, Notre Dame, Indiana. He was a Postdoctoral Research Affiliate at the Information Systems Laboratory, Stanford University in 1991. He was the 2001 Cor Wit Visiting Professor at the Delft University of Technology and had held visiting positions at Stanford University, and U.C. Berkeley.

Lang Tong is a Fellow of IEEE. He received the 1993 Outstanding Young Author Award from the IEEE Circuits and Systems Society, the 2004 best paper award (with Min Dong) from IEEE Signal Processing Society, and the 2004 Leonard G. Abraham Prize Paper Award from the IEEE Communications Society (with Parvathinathan Venkitasubramaniam and Srihari Adireddy). $\mathrm{He}$ is also a coauthor of seven student paper awards. He received Young Investigator Award from the Office of Naval Research.

Lang Tong's research is in the general area of statistical signal processing, wireless communications and networking, and information theory. He has served as an Associate Editor for the IEEE Transactions on Signal Processing, the IEEE Transactions on Information Theory, and IEEE Signal Processing Letters. He was named as a 2009-2010 Distinguished Lecturer by the IEEE Signal Processing Society. 